\title{
Squeezing and temperature measurement in Bose-Einstein Condensates
}

\author{
J. Rogel-Salazar and G.H.C. New \\ Quantum Optics 8 S Laser Science, Department of Physics, \\ Imperial College, London SW7 2BW, U.K. \\ S. Choi and K. Burnett \\ Clarendon Laboratory, Department of Physics, \\ University of Oxford, Parks Road, Oxford OX1 3PU, U.K.
}

\begin{abstract}
In this paper we discuss the presence of temperature-dependent squeezing in the collective excitations of trapped Bose-Einstein condensates, based on a recent theory of quasiparticle damping. A new scheme to measure temperature below the critical temperature $\left(T \ll T_{c}\right)$ is also considered.
\end{abstract}




\section{INTRODUCTION}

Following the first experimental observation of Bose-Einstein condensation in 1995 1], analogies between this phenomenon and non-linear optics have been pointed out [2, 3]. Recently, squeezing of the matter waves has been analysed [4, 5, 6]; by considering the role of the nonlinear interactions between the atoms in generating entangled atomic beams, namely the spin-exchanging collisions of spinor Bose-Einstein condensates (BEC's).

Here we analyse squeezing in relation to the coupling of collective excitations in a Bose condensed gas in a trap. Most of the observed effects of elementary excitations can be ex-

plained using the standard Bogoliubov theory [7]. However, it cannot account for higher order processes such as the Beliaev damping of the excitations observed recently for a scissors mode of a BEC in ${ }^{87} \mathrm{Rb}$ [8]. Beliaev damping is a mechanism in which a quasiparticle with energy $E_{2}$ interacts with the condensate and generates two quasiparticles with energy $E_{1}$. The process is actually based on a four-wave process that is desguised as a three-wave interaction analogous to degenerate parametric down-conversion. The new quasiparticles, being produced in pairs, constitute a squeezed twin phonon beam. Recent theoretical development including the presence of interactions between the quasiparticles [9] provides an explanation for such processes. Finding quantitative estimates for the amount of squeezing and entanglement based on this theory is one of the main tasks of this paper.

Considering a system containing $10000{ }^{87} \mathrm{Rb}$ atoms in a spherical trap, we calculate the degree of squeezing of the quasiparticle excitations. A related experimental challenge that we also address is the accurate determination of temperature in BEC's at $T \ll T_{c}$. The temperature is currently estimated by fitting a thermal Gaussian profile to the atomic cloud. We propose a different way to determine temperature by observing the variation of the envelope of collective oscillations.

The paper is structured as follows: In Section II the Hamiltonian in both particle and quasiparticle basis is discussed in relation to squeezing. The Hamiltonian in the quasiparticle basis accommodates processes such as the Landau and Beliaev damping. In Section III we obtain equations of motion for the quasiparticle modes and give quantitative estimates for the degree of squeezing and entanglement in the quasiparticle excitations. The expected "damping rate" at various temperatures is calculated. This is then used as a temperature measurement calibration for BEC. In Section IV we conclude. 


\section{MANY-BODY HAMILTONIAN}

Within the second quantised formalism, the many-body Hamiltonian for a system of bosons with binary interactions can be written as,

$$
\hat{H}=\sum_{i j} H_{i j}^{s p} \hat{a}_{i}^{\dagger} \hat{a}_{j}+\frac{1}{2} \sum_{i j k m}\langle i j|\hat{V}| k m\rangle \hat{a}_{i}^{\dagger} \hat{a}_{j}^{\dagger} \hat{a}_{k} \hat{a}_{m},
$$

where the matrix elements $H_{i j}^{s p}$ are given by

$$
H_{i j}^{s p}=\int d^{3} \mathbf{r} \psi_{i}^{*}(\mathbf{r}) \hat{H}^{s p} \psi_{j}(\mathbf{r}) .
$$

Here, $H^{s p}=-\frac{\hbar}{2 m} \nabla^{2}+V_{\text {trap }}$, is the single-particle Hamiltonian with a confining potential $V_{\text {trap }}$, and the basis state wave functions are $\psi_{i}(\mathbf{r})$. The matrix element $\langle i j|\hat{V}| k m\rangle$ denotes the matrix element for a bare interaction potential $\hat{V}(\mathbf{r})$ between two atoms; in this work, we shall consider the typical delta-function contact interaction potential for $\hat{V}(\mathbf{r})$. The operators $\hat{a}_{i}^{\dagger}$ and $\hat{a}_{i}$ are the creation and annihilation operators for mode $i$ that obey the Boson commutation relations

$$
\left[\hat{a}_{i}, \hat{a}_{j}^{\dagger}\right]=\delta_{i j}, \quad\left[\hat{a}_{i}, \hat{a}_{j}\right]=\left[\hat{a}_{i}^{\dagger}, \hat{a}_{j}^{\dagger}\right]=0
$$

The Hamiltonian (11) is written in a single-particle basis where the operator $\hat{a}_{i}$ annihilates a particle from the state with wave function $\psi_{i}(\mathbf{r})$. The wave function $\psi_{0}(\mathbf{r})$ describes the condensate, whilst the remaining functions form a complete set orthogonal to the condensate.

\section{A. Particle Hamiltonian}

Let us first investigate the condensate by concentrating only on the condensate mode. This one-mode approximation applies well for temperatures $T \simeq 0$ which is indeed the case for many experiments. We find the Hamiltonian is then simplified to

$$
\hat{H}=\epsilon_{00} \hat{a}_{0}^{\dagger} \hat{a}_{0}+\frac{1}{2}\langle 00|V| 00\rangle \hat{a}_{0}^{\dagger} \hat{a}_{0}^{\dagger} \hat{a}_{0} \hat{a}_{0} .
$$

Treating the deviations from the ideal condensate as perturbations, we are able to expand $\hat{a}_{0}$ as

$$
\hat{a}_{0}=z+\hat{c}
$$

where $z=\sqrt{N_{0}}$ represents the mean-field (condensate), and $\hat{c}$ is the small quantum perturbation. $\hat{c}$ may take the meaning of non-condensed atoms. We choose the phase so that 
$z$ is real. The mean-field approximation, although not exact, has been very successful in describing a large number of experimental results. Using the expansion for $\hat{a}_{0}$ and splitting our Hamiltonian into a Gaussian and a non-Gaussian parts, we get the Gaussian part of our Hamiltonian as

$$
\hat{H}=\epsilon_{0}\left[z^{2}+z\left(\hat{c}+\hat{c}^{\dagger}\right)+\hat{c}^{\dagger} \hat{c}\right]+\frac{\langle 00|V| 00\rangle}{2}\left[z^{4}+z^{2}\left(\hat{c}^{2}+\hat{c}^{\dagger 2}\right)+2 z^{3}\left(\hat{c}+\hat{c}^{\dagger}\right)+4 z^{2} \hat{c}^{\dagger} \hat{c}\right] .
$$

The non-Gaussian part may be treated using perturbation theory [10]. The resulting Heisenberg equations of motion for $\hat{c}$ and $\hat{c}^{\dagger}$ are

$$
\left(\begin{array}{c}
\dot{\hat{c}}(t) \\
\dot{\grave{c}}(t)
\end{array}\right)=\mathrm{i}\left(\begin{array}{rr}
-\mathrm{a} & -\mathrm{b} \\
\mathrm{b} & \mathrm{a}
\end{array}\right)\left(\begin{array}{c}
\hat{c}(t) \\
\hat{c}^{\dagger}(t)
\end{array}\right)+\mathrm{i}\left(\begin{array}{r}
-\kappa \\
\kappa
\end{array}\right),
$$

where $\mathrm{a}=\frac{1}{\hbar}\left(\epsilon_{0}+2\langle 00|V| 00\rangle z^{2}\right), \mathrm{b}=\frac{1}{\hbar}\langle 00|V| 00\rangle z^{2}$, and $\kappa=\frac{1}{\hbar}\left(\epsilon_{0} z+\langle 00|V| 00\rangle z^{3}\right)$. The solutions of these equations are

$$
\left(\begin{array}{c}
\hat{c}(t) \\
\hat{c}^{\dagger}(t)
\end{array}\right)=\left(\begin{array}{cc}
A & B \\
B^{*} & A^{*}
\end{array}\right)\left(\begin{array}{c}
\hat{c}(0) \\
\hat{c}^{\dagger}(0)
\end{array}\right)+\left(\begin{array}{c}
D \\
D^{*}
\end{array}\right),
$$

where $A=\cos \Upsilon t-\mathrm{i} \frac{\mathrm{a}}{\Upsilon} \sin \Upsilon t, B=-\mathrm{i} \frac{\mathrm{b}}{\Upsilon} \sin \Upsilon t, D=\frac{(\mathrm{a}-\mathrm{b}) \kappa}{\Upsilon^{2}} \cos \Upsilon t-\mathrm{i} \frac{\kappa}{\Upsilon} \sin \Upsilon t+\frac{(\mathrm{b}-\mathrm{a}) \kappa}{\Upsilon^{2}}$, and $\Upsilon=\sqrt{\mathrm{a}^{2}-\mathrm{b}^{2}}$. It is important to note that the above equations are formally equivalent to dynamic equations that describe parametric down conversion in quantum optics [11] if $\kappa \equiv 0$.

Defining the quadrature operators as $\hat{X}_{p}(t)=\frac{\hat{c}(t)+\hat{c}^{\dagger}(t)}{2}$ and $\hat{Y}_{p}(t)=\frac{\hat{c}(t)-\hat{c}^{\dagger}(t)}{2 \mathrm{i}}$, we have that the variances can be written as

$$
\begin{aligned}
\left(\Delta \hat{X}_{p}(t)\right)^{2} & =\frac{(2 \tilde{n}+1)}{4}\left(\cos ^{2} \Upsilon t+\frac{(\mathrm{a}-\mathrm{b})^{2}}{\Upsilon^{2}} \sin ^{2} \Upsilon t\right) \\
\left(\Delta \hat{Y}_{p}(t)\right)^{2} & =\frac{(2 \tilde{n}+1)}{4}\left(\cos ^{2} \Upsilon t+\frac{(\mathrm{a}+\mathrm{b})^{2}}{\Upsilon^{2}} \sin ^{2} \Upsilon t\right)
\end{aligned}
$$

where $\tilde{n}$ is the number of excited particles. In this case, the magnitude of a and $\mathrm{b}$, or equivalently the magnitude and phase of the mean field, $z$, control the amount of squeezing of the particule quadrature operators.

\section{B. Quasiparticle Hamiltonian}

On the other hand, it is possible to describe the system including the higher order processes. The dominant ones correspond to the quadratic Hamiltonian $\hat{H}_{Q}=\hat{H}_{0}+\hat{H}_{1}+\hat{H}_{2}$, 
where the subscripts $0,1,2$ indicate the order in $\hat{a}_{i}$. This may be diagonalised exactly using the standard Bogoliubov transformations, changing the Hamiltonian to a quasiparticle basis where the quasiparticle operators $\hat{\beta}_{i}$ are defined by ${ }^{1}$

$$
\beta_{i}=\sum_{j \neq 0} U_{i j}^{*} a_{j}-V_{i j}^{*} a_{j}^{\dagger}
$$

Here $U_{i j}$ and $V_{i j}$ are the well-known matrices associated with the Bogoliubov transformations, obeying the orthogonality and symmetry conditions $U U^{\dagger}-V V^{\dagger}=1$ and $U V^{T}-V U^{T}=0$. These matrices have to be evaluated numerically depending on temperature and the geometry of the trap. The non-quadratic terms are expected to be small and can be dealt with perturbatively. The energy and shape of the condensate change when one includes these terms. It has been shown [9] that in terms of the quasiparticle operator $\beta_{i}$ the effective Hamiltonian can be written as

$$
H^{\prime}=\mathrm{const}+\sum_{i \neq 0}\left(\epsilon_{i}+\Delta \epsilon_{i}\right) \beta_{i}^{\dagger} \beta_{i}+\left\{\left[\sum_{i j k \neq 0}\left[\xi_{i j k} \beta_{i} \beta_{j} \beta_{k}+\zeta_{i j k} \beta_{i}^{\dagger} \beta_{j} \beta_{k}\right]+\sum_{i \neq 0} \eta_{i} \beta_{i}\right]+\text { h.c. }\right\}
$$

where the const term simply shifts the zero of energy and $\Delta \epsilon_{i}$ is the energy shift from first order perturbation theory. The Hamiltonian given by equation (11) contains terms beyond the Bogoliubov approximation, showing that we are treating interacting quasiparticles. We are thus taking into account important processes such as Landau and Beliaev damping. Landau processes, in which two quasiparticles collide to form a single quasiparticle, can not occur at zero temperature because there are no excited quasiparticles. However, they are dominant at high temperatures. Expressions for the coefficients of equation (11) are given in Appendix A. As we are interested in finite temperature, the $U$ and $V$ matrices are calculated self-consistently using the Bogoliubov-de Gennes (BdG) formalism for each temperature. From the Hamiltonian (11), the Heisenberg equation of motion for $\beta_{p}$ is

$$
\mathrm{i} \dot{\beta}_{p}=\frac{\eta_{p}^{*}}{\hbar}+\omega_{p} \beta_{p}+\sum_{j, k \neq 0} \sigma_{j k} \beta_{j} \beta_{k}+\sum_{j, k \neq 0} \rho_{j k} \beta_{j}^{\dagger} \beta_{k}^{\dagger}+\sum_{j, k \neq 0} \nu_{j k} \beta_{k}^{\dagger} \beta_{j}
$$

\footnotetext{
${ }^{1}$ For simplicity from now on we drop the explicit notation in the operators
} 
where

$$
\begin{aligned}
\omega_{p} & =\left(\epsilon_{p}+\Delta \epsilon_{p}\right) / \hbar \\
\sigma_{j k} & =\zeta_{p j k} / \hbar \\
\rho_{j k} & =\left(\xi_{p j k}^{*}+\xi_{j k p}^{*}+\xi_{k p j}^{*}\right) / \hbar, \\
\nu_{j k} & =\left(\zeta_{j p k}^{*}+\zeta_{j k p}^{*}\right) / \hbar .
\end{aligned}
$$

Equation (12) and its complex conjugate are valid for the general case. However, a dominant process can be defined by choosing the appropriate geometry for the trap. In this form, the number of states involved is reduced [12]. The trap geometry can be modified by adjusting the frequencies in the radial and axial directions independently and the selection of a dominant mode in this way has been demonstrated [13]. In particular, the observation of a Beliaev process has recently been reported for a scissors mode, where one mode is resonantly coupled to two modes of half the original frequency [8]; this is the kind of process that we seek to model in the present paper. We note that terms in $\xi$ and $\eta$ describe the spontaneous decay of quasiparticles and thus do not generally conserve energy. Therefore, we will only keep terms containing $\sigma$ and $\nu$. More details of these arguments can be found in reference [14], and the main points are summarised in Appendix B. For a Beliaev process, the equations of motion for modes $p=1$ and $p=2$ are

$$
\begin{aligned}
& \dot{\beta}_{1}=-\mathrm{i} \omega_{1} \beta_{1}-\mathrm{i} \nu_{21} \beta_{2} \beta_{1}^{\dagger}, \\
& \dot{\beta}_{2}=-\mathrm{i} \omega_{2} \beta_{2}-\mathrm{i} \frac{\nu_{21}}{2} \beta_{1} \beta_{1} .
\end{aligned}
$$

plus their complex conjugates. The factor of 2 that appears in equation (14) comes from the fact that $\sigma_{11}=\nu_{21} / 2$.

Considering the system at a temperature $T=20 \mathrm{nK}$, with $N=10000$ particles and a trap frequency $\omega_{\text {trap }}=2 \pi \times 100 \mathrm{~Hz}$ with spherical geometry and using ${ }^{87} \mathrm{Rb}$ atoms for which the s-wave scattering length $a=10 a_{0}$, where $a_{0}$ is the Bohr radius, the coefficient $\nu_{21}$ equals $1.90 \times 10^{-2}$ in trap units. The equations of motion for $\beta_{1}$ and $\beta_{1}^{\dagger}$ are profoundly affected by temperature through the values of $\nu_{21}$.

Equations of this sort have extensively been studied in quantum optics. In this case we apply them in order to study squeezing in the quasiparticle excitations and its temperature 
dependence. It is important to mention that the damping of excitations has earlier been described in terms of nonlinear mixing [15]; this work differs from that description as the operator nature of the quasiparticle annihilation operator $\beta_{i}$ is retained, and the calculation is not restricted to the quadratic approximation. We are in effect extending the work of Ref. 15], such that spontaneous quantum processes are included.

\section{TEMPERATURE DEPENDENT COUPLING PROCESS}

\section{A. Non-depleted regime}

In the case in which mode 2 has a much larger population than mode 1 , we may approximate the operator $\beta_{2}$ by a c-number $b_{2}$ and ignore the depletion of mode 2 . The solutions

of the Heisenberg equations of motion for the operators $\beta_{1}$ and $\beta_{1}^{\dagger}$ are then given by

$$
\left(\begin{array}{l}
\beta_{1}(t) \\
\beta_{1}^{\dagger}(t)
\end{array}\right)=\left(\begin{array}{rr}
\cosh (\Omega t) & -\mathrm{i} \sinh (\Omega t) \\
\mathrm{i} \sinh (\Omega t) & \cosh (\Omega t)
\end{array}\right)\left(\begin{array}{l}
\beta_{1}(0) \\
\beta_{1}^{\dagger}(0)
\end{array}\right),
$$

where $\Omega=\nu_{21} b_{2}$, and we have chosen the phases such that the coefficients are real. This represents the physical situation where the pump is continuously driven by a resonant excitation. It is important to mention that the approximation is no longer valid when a noticeable down-conversion has occurred.

Solutions (16) are equivalent to equations obtained in the description of degenerate parametric down-conversion in quantum optics. This process is well-known to be an efficient source of squeezed states. In this case, the corresponding squeezing parameter is

$$
\tau=\Omega t
$$

which is temperature dependent via $\Omega$.

Analogously, one can define the quadrature operators for $\beta$ and $\beta^{\dagger}$ and calculate the variances

$$
\begin{aligned}
& (\Delta X(t))^{2}=\frac{\left(2 N_{1}+1\right)}{4} \exp (-2 \Omega t), \\
& (\Delta Y(t))^{2}=\frac{\left(2 N_{1}+1\right)}{4} \exp (2 \Omega t)
\end{aligned}
$$


where $N_{1}$ is the number of particles in mode 1 . In optics the quadrature operators are well-defined quantities corresponding to the amplitude and the phase of the electromagnetic (EM) oscillation. In a similar fashion, we give our quadrature operators $X$ and $Y$ the interpretations of amplitude and phase of oscillations. The dependence of equations (18) and (19) implies that the amount of squeezing is related to the number of lower-mode atoms present. Consequently, it is connected to the temperature of the ultracold atoms in the condensate [16], defined in terms of the initial Bose-Einstein (BE) distribution of quasiparticles.

However, in an experiment what one measures some correlation functions, for instance $\left\langle\beta_{1}^{\dagger} \beta_{1}\right\rangle$. Assuming an initial number state in the quasiparticle basis, some important correlation functions are given by:

$$
\begin{aligned}
& \left\langle\beta_{1}(t) \beta_{1}(t)\right\rangle=-\mathrm{i}\left(N_{1}+\frac{1}{2}\right) \sinh (2 \Omega t), \\
& \left\langle\beta_{1}^{\dagger}(t) \beta_{1}(t)\right\rangle=\left(N_{1}+\frac{1}{2}\right) \cosh (2 \Omega t)-\frac{1}{2} .
\end{aligned}
$$

A plot of the correlation function (20) is shown in Figure 2 a). A non-zero value for this correlation function implies the presence of squeezing and hence of entanglement between the two modes. The quantity given by equation (21) carries information about the behaviour of the population in mode 1 and it is directly measured as the amplitude of oscillation. A plot of this correlation function as a function of time $(t)$ and temperature $(T)$ is given in Figure $2 \mathrm{~b}$ ). At $t=0$ the behaviour is determined by the BE distribution of $N_{1}$. As time evolves the population of the lower mode increases.

\section{B. Depleted regime}

Let us now include the effect that depletion of mode 2 has in the description of the coupling process. In order to tackle the problem, we will consider the equations of motion for the number operators $N_{i}=\beta_{i}^{\dagger} \beta_{i}$. They can be calculated through the Heisenberg equations (13] 14), namely

$$
\begin{aligned}
& \frac{\mathrm{d} N_{1}}{\mathrm{~d} t}=\mathrm{i} \nu_{21}\left(\beta_{2}^{\dagger} \beta_{1} \beta_{1}-\beta_{1}^{\dagger} \beta_{1}^{\dagger} \beta_{2}\right) \\
& \frac{\mathrm{d} N_{2}}{\mathrm{~d} t}=\mathrm{i} \frac{\nu_{21}}{2}\left(\beta_{2} \beta_{1}^{\dagger} \beta_{1}^{\dagger}-\beta_{2}^{\dagger} \beta_{1} \beta_{1}\right) .
\end{aligned}
$$


It is possible to uncouple the equations (22) and (23) using the constant of motion $A=N_{1}+2 N_{2}$ and calculating the second derivative. The uncoupled equations are given by

$$
\begin{aligned}
& \frac{\mathrm{d}^{2} N_{1}}{\mathrm{~d} t^{2}}=\nu_{21}^{2}\left(-3 N_{1}^{2}+2 A N_{1}+A\right), \\
& \frac{\mathrm{d}^{2} N_{2}}{\mathrm{~d} t^{2}}=\frac{\nu_{21}^{2}}{2}\left(12 N_{2}^{2}-8 A N_{2}+A^{2}-A\right) .
\end{aligned}
$$

When the population of mode 2 is large, it is possible to determine the time evolution by taking the average of equation (25). In that case we have a c-number second-order differential equation with the following initial conditions

$$
\begin{aligned}
\left.\frac{\mathrm{d} N_{2}}{\mathrm{~d} t}\right|_{t=0} & =0 \\
N_{2}(0) & =N_{20} ;
\end{aligned}
$$

The solution can be expressed as follows

$$
N_{2}(t)= \begin{cases}N_{20}+\left(\alpha_{2}-N_{20}\right) \operatorname{sn}^{2}\left(\frac{\nu_{21}}{2} \sqrt{\frac{\alpha_{1}-N_{20}}{6}} t, \sqrt{\frac{\alpha_{2}-N_{20}}{\alpha_{1}-N_{20}}}\right), & \text { for } N_{20}<\alpha_{2}, \\ \alpha_{1}+\left(N_{20}-\alpha_{1}\right) \operatorname{nd}^{2}\left(\frac{\nu_{21}}{2} \sqrt{\frac{\alpha_{1}-\alpha_{2}}{6}} t, \sqrt{\frac{N_{20}-\alpha_{2}}{\alpha_{1}-\alpha_{2}}}\right), & \text { for } N_{20}>\alpha_{2},\end{cases}
$$

where $\operatorname{sn}(u, k)$ and $\operatorname{nd}(u, k)$ are the standard Jacobi elliptic functions [18], and the coefficient $\alpha_{1}$ and $\alpha_{2}$ are the roots of the quadratic polynomial $P\left(N_{2}\right)=-N_{2}^{2}+\left(A-N_{20}\right) N_{2}-N_{20}^{2}-$ $\left(A^{2}-A\right) / 4+N_{20} A$. It has been already mentioned that the coefficient $\nu_{21}$ is temperature dependent; therefore the population will change accordingly. The initial population was calculated for different temperatures as prescribed by the BE distribution. An initial driving was also taken into account. The solution was then calculated using these parameters; a plot is shown in Figure 3 as a function of temperature and time.

The connection between the theory and experiment can be accomplished by observing that the initial part of the population surface can be fitted by a curve of the form $A_{1} \cos (\Gamma t)$. The parameter $\Gamma$ is related to the solution (28) as

$$
\Gamma=\nu_{21} \sqrt{\frac{\left(\alpha_{1}-N_{20}\right)\left(N_{20}-\alpha_{2}\right)}{12 N_{20}}} .
$$

Following this line of analysis, a fitting routine was used to obtain a plot of $\Gamma$ as a function of temperature (Figure 4). We can see from the plot that for lower temperatures the influence of the initial population is preeminent. For higher temperatures the coupling coefficient $\nu_{21}$ 
dwindles quickly, thus the effect of the initial population is hidden. It can clearly be seen that there is a competition between the initial population and the coupling coefficient. The quantity $\Gamma$ is notable in that it is a readily measurable temperature dependent quantity that links to the squeezing of quasiparticles.

\section{DISCUSSION}

The detection of squeezed states in the case of the electromagnetic field can be achieved through well-known techniques, e.g. homodyne detection. On the other hand, scattering experiments have also been proposed as a way to detect squeezing in bosons [17]. The proposal makes use of a particle scattering off a boson field. Provided the particle is prepared in the proper input state, it can either absorb or emit a boson, and these two scattering processes can coherently interfere when the boson field is in a squeezed state. The change in the rate of scattering into a particular state gives a signature that the field has been squeezed. A schematic setup for the detection of squeezing is shown in Figure 5 . An incoming beam of frequency $\omega_{-}$is divided into two by a beam splitter, with one of the beams frequency shifted to $\omega_{+}$, which may be achieved through acusto-optic modulation. The two beams are made incident into the boson field, e.g. BEC. Particles scattered off the squeezed field are detected by the counter. This would provide an experimentally feasible way to directly measure squeezing in BEC.

In conclusion, we have described a process analogous to parametric down-conversion in trapped Bose-Einstein condensates. A fully second quantised order theory has been used to depict the condensate at finite temperature. The process corresponds to Beliaev damping, where a quantum of higher energy is divided into two quanta of lower energy. The coupling process is temperature dependent; this implies that any amount of squeezing in the elementary excitations of the condensate would give a direct indication of the temperature.

\section{Acknowledgments}

We would like to thank Dr. M. Rusch for helpful discussions. This work has been

supported by CONACyT, EPSRC, Royal Commission for the Exhibition of 1851 and the EU. 


\section{APPENDIX A}

The coefficients that appear in equation (11) written in the position representation (using the contact potential) are determined by

$$
\begin{aligned}
\xi_{i j k}= & \sqrt{N_{0}} U_{0} \int \mathrm{d}^{3} \mathbf{r}\left[\psi_{0}^{*}(\mathbf{r}) v_{i}(\mathbf{r}) u_{j}(\mathbf{r}) u_{k}(\mathbf{r})+\psi_{0}(\mathbf{r}) u_{i}(\mathbf{r}) v_{j}(\mathbf{r}) v_{k}(\mathbf{r})\right] \\
\zeta_{i j k}= & \sqrt{N_{0}} U_{0} \int \mathrm{d}^{3} \mathbf{r}\left\{\psi_{0}^{*}(\mathbf{r})\left[u_{i}^{*}(\mathbf{r}) u_{j}(\mathbf{r}) u_{k}(\mathbf{r})+v_{i}^{*}(\mathbf{r}) v_{j}(\mathbf{r}) u_{k}(\mathbf{r})+v_{i}^{*}(\mathbf{r}) u_{j}(\mathbf{r}) v_{k}(\mathbf{r})\right]\right. \\
& \left.+\psi_{0}\left[u_{i}^{*}(\mathbf{r}) u_{j}(\mathbf{r}) v_{k}(\mathbf{r})+u_{i}^{*}(\mathbf{r}) v_{j(\mathbf{r})} u_{k}(\mathbf{r})+v_{i}^{*}(\mathbf{r}) v_{j}(\mathbf{r}) v_{k}(\mathbf{r})\right]\right\} \\
\eta_{i}= & -\sum_{q \neq 0}\left(\zeta_{q q i}+\zeta_{q i q}\right) N_{q}
\end{aligned}
$$

where the indices $i, j$ and $k$ are labels that denote eigen-energy levels. For a 3-D condensate, the level $i$ is an implicit notation for the quantum numbers $n, l$ and $m$.

\section{APPENDIX B}

To prove that Beliaev damping is indeed the dominant process with the given trap geometry discussed in the paper, it is only necessary to calculate various contributions from the framework of second order perturbation theory. The energy shift due to various higher order terms is given by the expression

$$
\begin{aligned}
\Delta E(p)= & -\sum_{i j \neq 0} \frac{\left|\xi_{p i j}^{P 3}\right|^{2}}{2\left(\epsilon_{p}+\epsilon_{i}+\epsilon_{j}\right)}\left[1+N_{i}+N_{j}\right] \\
& +\sum_{i j \neq 0} \frac{\left|\zeta_{p i j}+\zeta_{p j i}\right|^{2}}{2\left(\epsilon_{p}-\epsilon_{i}-\epsilon_{j}\right)}\left[1+N_{i}+N_{j}\right] \\
& +\sum_{i j \neq 0} \frac{\left|\zeta_{i j p}+\zeta_{i p j}\right|^{2}}{\epsilon_{p}-\epsilon_{i}+\epsilon_{j}}\left[N_{j}-N_{i}\right],
\end{aligned}
$$

where line (B1a) indicates spontaneous decay of quasiparticles. Here the coefficient $\xi_{p i j}^{P 3}$ is defined as a sum over permutations of the three indices in $\xi_{p j k}$, i.e. $\xi_{p i j k}^{P 3}=\xi_{p j k}+\xi_{p k j}+\xi_{j p k}+$ $\xi_{j k p}+\xi_{k p j}+\xi_{k j p}$. Lines (B1b) and (B1c) give Beliaev and Landau damping processes respectively. Typical values are given in Ref. [14] and owing to the resonance in the denominator, 
(B1b) may be made dominant with a correct choice of experimental parameters [13].

[1] M. H. Anderson, J. R. Enser, M. R. Matthews, C. E. Wieman and E. Cornell. Science 269, $198(1995)$.

[2] L. Deng, E. W. Hagley, J. Wen, M. Trippenbach, Y. Band, P. S. Julienne, J. E. Simsarian, K. Helmerson, S. L. Rolston and W.D. Phillips, Nature 398, 218 (1999).

[3] W. P. Reinhardt and C. W. Clark, J. Phys. B. 30 L785 (1997).

[4] L. M. Duan, A. Sørensen, J. I. Cirac, P. Zoller, Phys. Rev. Lett. 85, 3991 (2000).

[5] A. Sørensen, L. M. Duan , J. I. Cirac, P. Zoller, Nature 409, 63 (2001).

[6] H. Pu and P. Meystre, Phys. Rev. Lett. 85, 3987 (2000).

[7] N. Bogoliubov, J. Phys. 11, 23 (1947).

[8] E. Hodby, O. M. Maragò, G. Hechenblaikner and C. J. Foot, Phys. Rev. Lett. 86, 2196 (2001).

[9] S. A. Morgan, DPhil Thesis, University of Oxford (1999).

[10] J.A. Dunningham, M.J. Collett, D.F. Walls. Phys. Lett. A. 245, 49 (1998).

[11] S. Barnett and P.M. Radmore, Methods in Theoretical Quantum Optics. Oxford Science Publications (1997).

[12] E. Merzbacher, Quantum Mechanics. John Wiley \& Sons, INC. Third ed. (1998).

[13] G. Hechenbleiker, O. M. Maragò, E. Hodby, J. Arlt, S. Hopkins and C. J. Foot, Phys. Rev. Lett. 85, 692 (2000).

[14] M. Rusch, DPhil Thesis, University of Oxford (2000).

[15] S. A. Morgan, S. Choi, K. Burnett and M. Edwards, Phys. Rev. A 57, 3818 (1998).

[16] R. J. Marshall, G. H. C. New, K. Burnett, S. Choi. Phys. Rev. A 59, 2085 (1999).

[17] B. Yurke, Phys. Rev. Lett. 60, 2476 (1988).

[18] P. F. Byrd, M.D. Friedman, Handbook of Elliptic Integrals for Engineers and Scientist. Springer-Verlag, Second ed. (1971).

[19] H. Palevsky, K. Otnes, K.E. Larsson, Phys. Rev. 112, 11, (1958).

[20] D.G. Henshaw, A.D.B. Woods, Phys. Rev. 121, 1266, (1961).

[21] J. Stenger, S. Inouye, A.P. Chikkatur, D.M. Stamper-Kurn, D.E. Pritchard, W. Ketterle, Phys. Rev. Lett. 82, 4569 (1999). 


\section{Figure Captions}

1. Schematic diagram of energy levels for a BEC in a trap. In the Beliaev process, a quasiparticle of frequency $\omega_{2}$ interacts with the ground state generating two quasiparticles of frequency $\omega_{1}$ that divide the initial energy equally. This process is analogous to the optical parametric down-conversion.

2. a) Surface plot of the average $\left\langle\beta_{1} \beta_{1}\right\rangle$. A non-zero value of this quantity implies the presence of squeezing and hence entanglement. b) The average $\left\langle\beta_{1}^{\dagger} \beta_{1}\right\rangle$ describes the behaviour of the population in mode 1 . This solution is valid within the non-depleted regime. As discussed before, the behaviour at $t=0$ is determined by the BE distribution function.

3. The population $N_{2}$ as a function of temperature and time.

4. Plot of $\Gamma$ against temperature. This parameter is obtained by fitting a curve of the form $A_{1} \cos (\Gamma t)$ corresponds to the initial part of the envelope of the population $N_{2}$ shown in figure 3 .

5. Schematic figure for the detection of squeezing by particle scattering. 


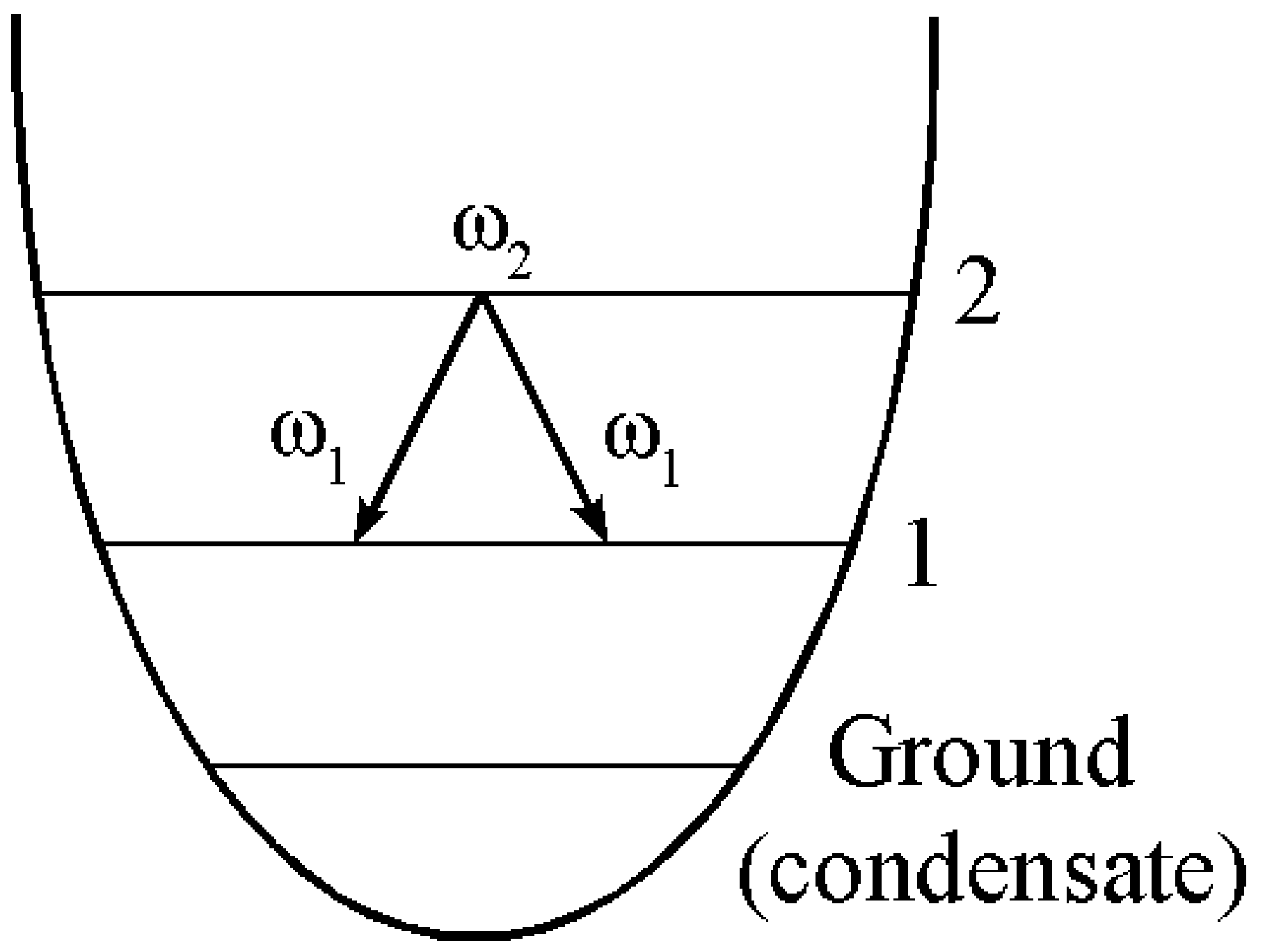


a) $\left\langle\beta_{1} \beta_{1}\right\rangle$

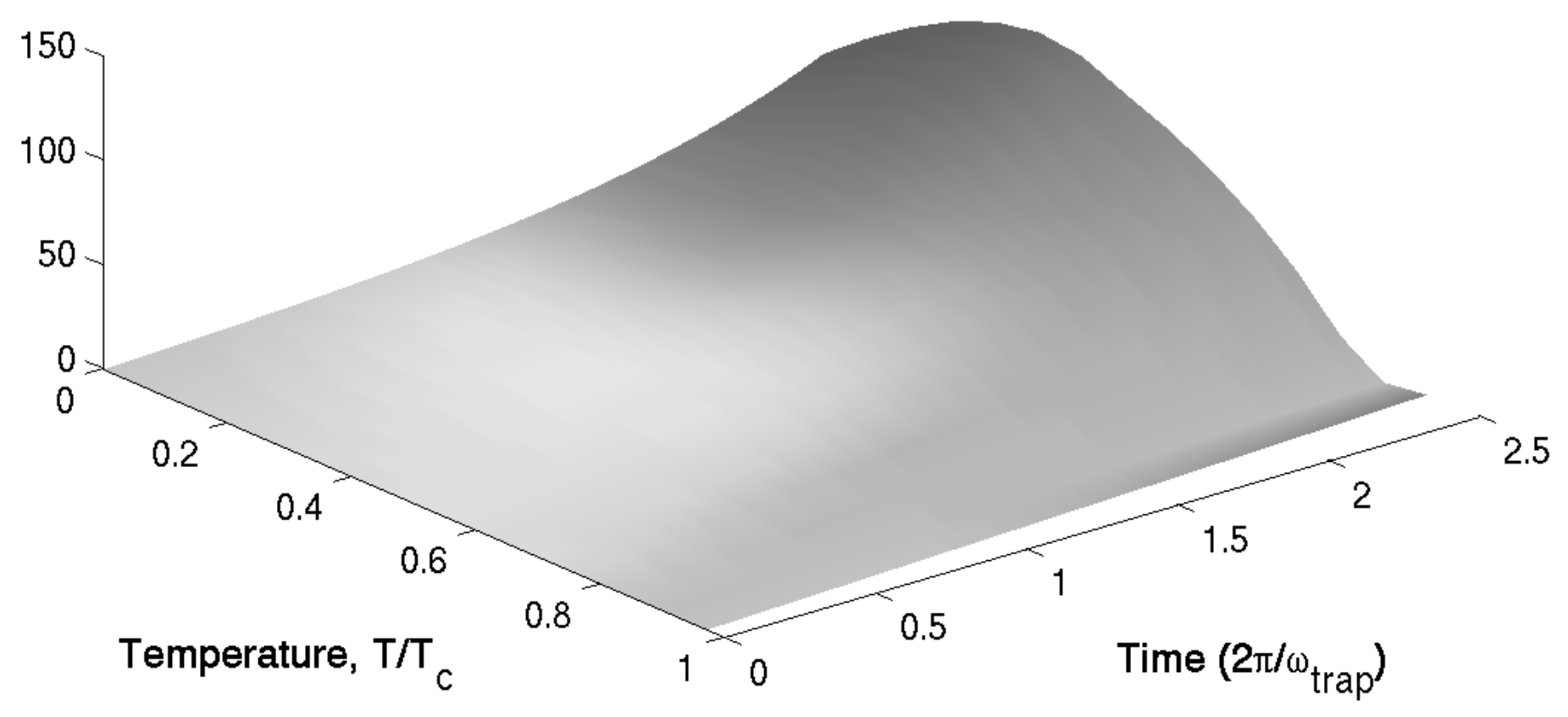

b) $\left\langle\beta_{1}^{+} \beta_{1}\right\rangle$

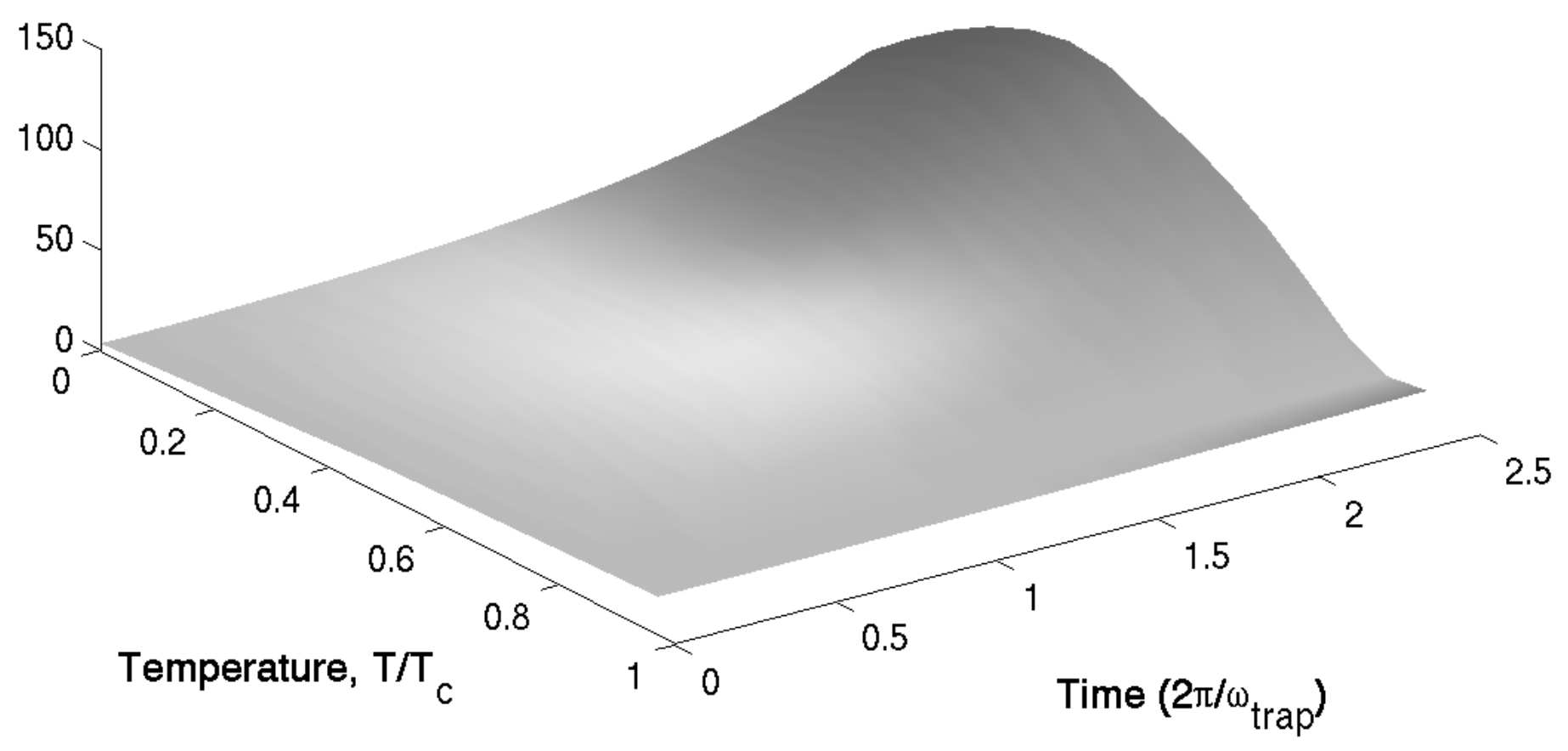




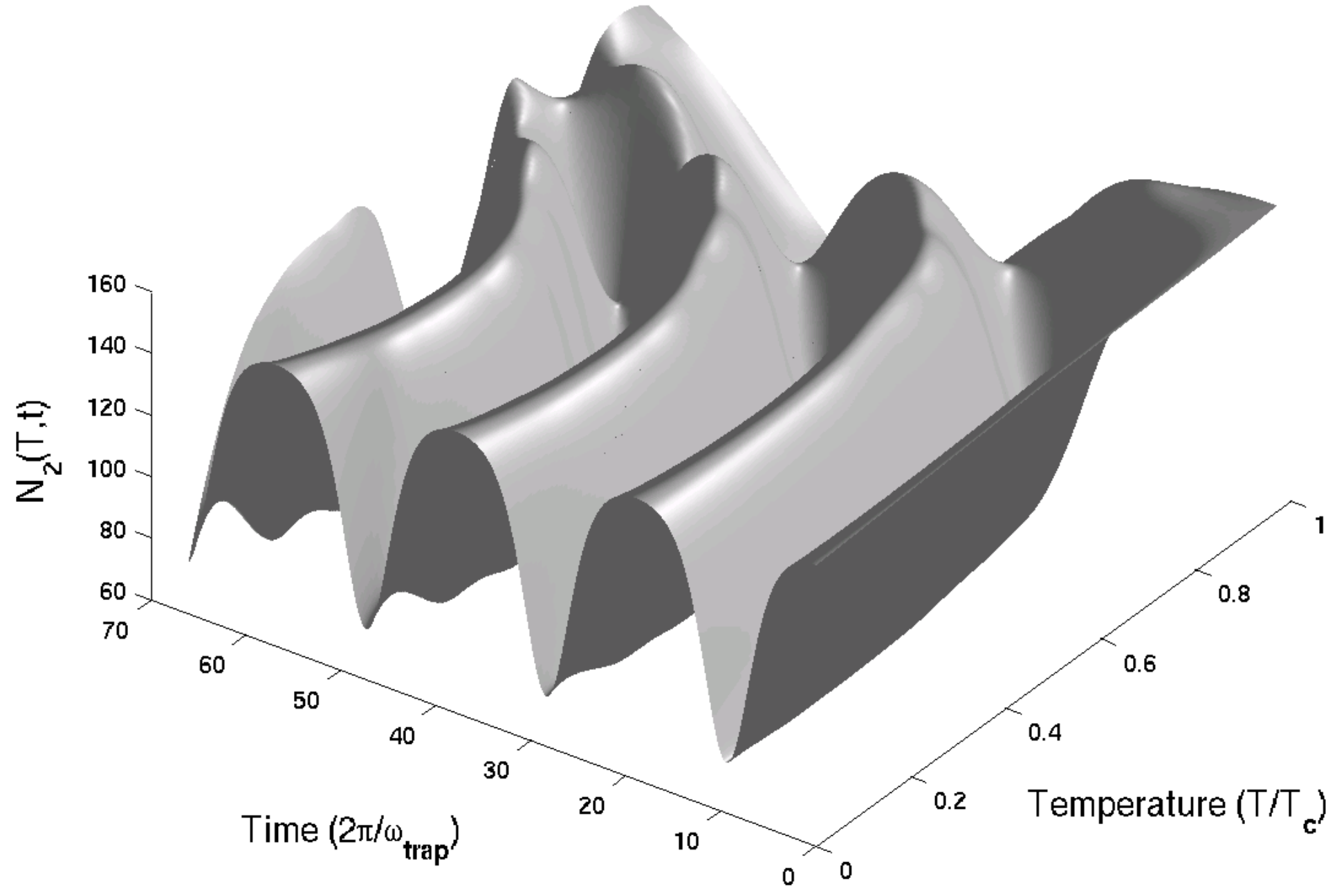




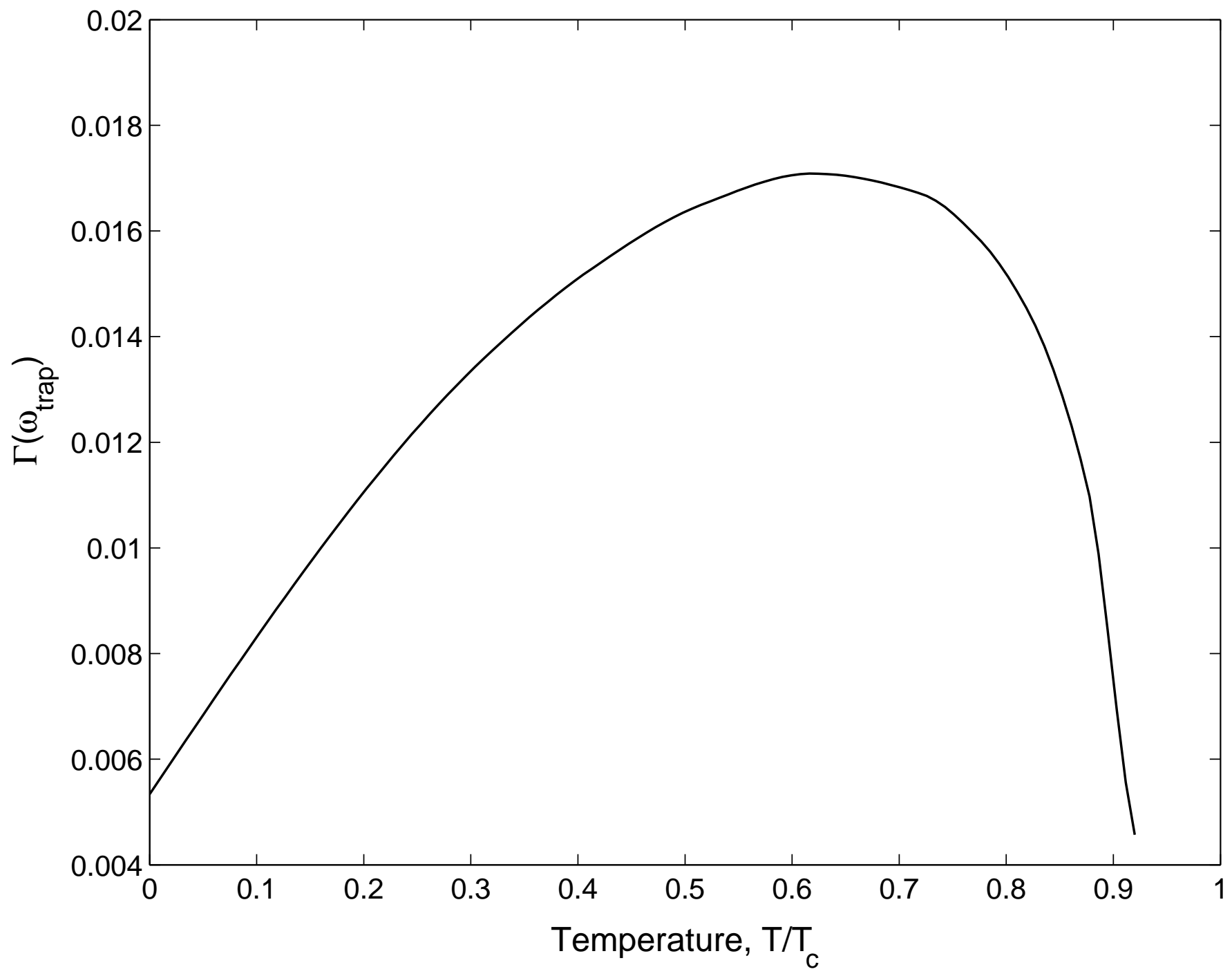




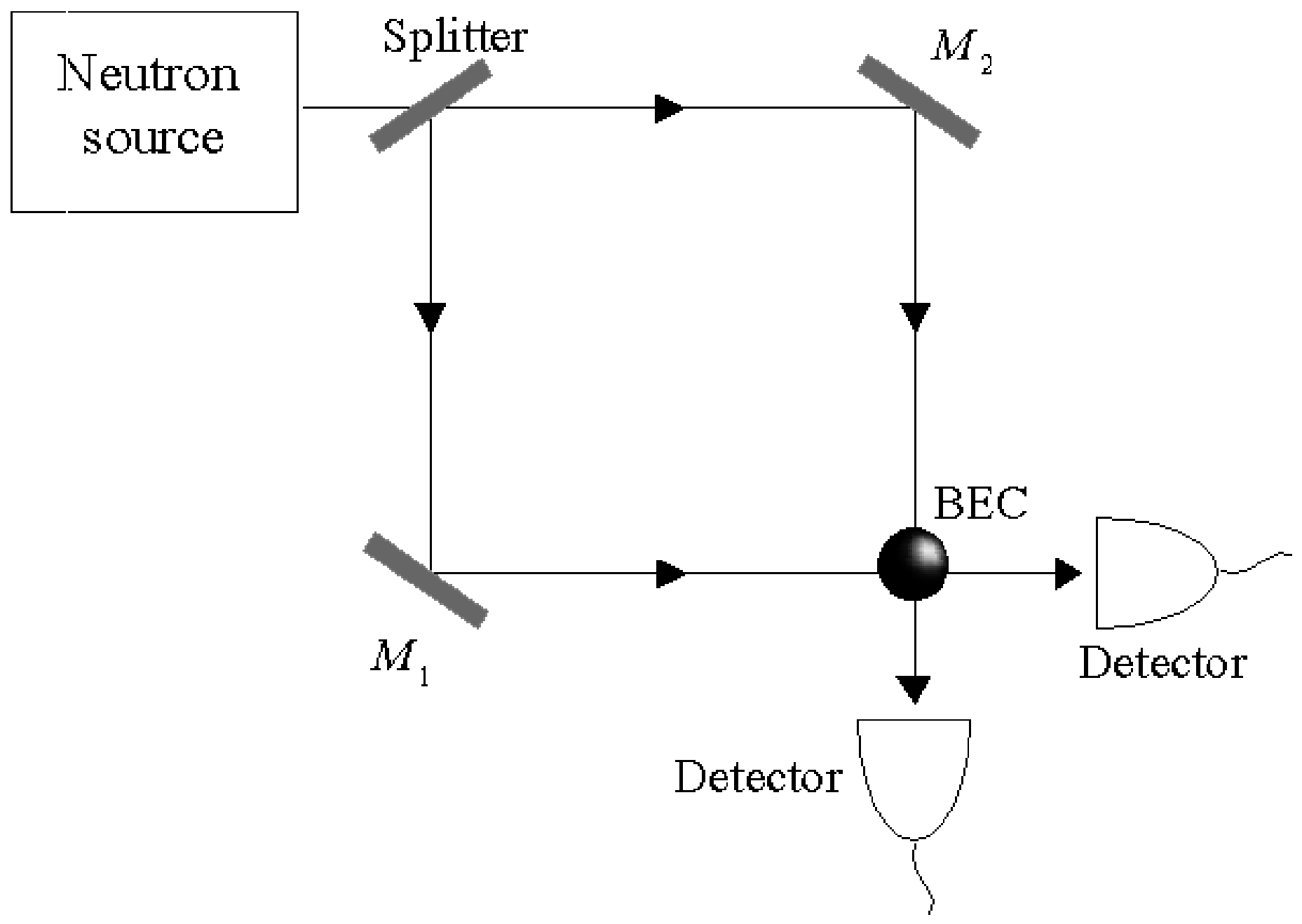

